Acta Crystallographica Section E

Structure Reports

Online

ISSN 1600-5368

\section{[Bis(diphenylphosphanyl)dimethylsilane- $\kappa^{2} P, P^{\prime}$ ]tetracarbonylchromium(0)}

\section{Normen Peulecke,* Stephan Peitz, Bernd H. Müller, Anke Spannenberg and Uwe Rosenthal}

Leibniz-Institut für Katalyse e. V. an der Universität Rostock, Albert-Einstein-Strasse 29a, 18059 Rostock, Germany

Correspondence e-mail: normen.peulecke@catalysis.de

Received 21 October 2010; accepted 26 October 2010

Key indicators: single-crystal X-ray study; $T=200 \mathrm{~K}$; mean $\sigma(\mathrm{C}-\mathrm{C})=0.002 \AA$; $R$ factor $=0.026 ; w R$ factor $=0.065 ;$ data-to-parameter ratio $=19.4$.

The title compound, $\left[\mathrm{Cr}\left(\mathrm{C}_{26} \mathrm{H}_{26} \mathrm{P}_{2} \mathrm{Si}\right)(\mathrm{CO})_{4}\right]$, was obtained by the reaction of $\mathrm{Ph}_{2} \mathrm{PSiMe}_{2} \mathrm{PPh}_{2}$ with $\mathrm{Cr}(\mathrm{CO})_{6}$ in refluxing toluene by ligand exchange. The $\mathrm{CrC}_{4} \mathrm{P}_{2}$ coordination geometry at the $\mathrm{Cr}$ atom is distorted octahedral, with a $\mathrm{P}$ $\mathrm{Cr}-\mathrm{P}$ bite angle of $80.27(1)^{\circ}$.

\section{Related literature}

For the synthesis of $\mathrm{Ph}_{2} \mathrm{PSiMe}_{2} \mathrm{PPh}_{2}$, see: Hassler \& Seidl (1988). The molecular and crystal structures of the tetracarbonyl tungsten complex of $\left[\left({ }^{i} \mathrm{Pr}_{2} \mathrm{~N}\right)_{2} \mathrm{BP}(\mathrm{H})\right]_{2} \mathrm{SiMe}_{2}$ and the tetracarbonyl molybdenum complex of $\left(\mathrm{PhPHSiMe}_{2}\right)_{2}$ were presented by Chen et al. (1999) and Sheldrick \& Borkenstein (1977), respectively.

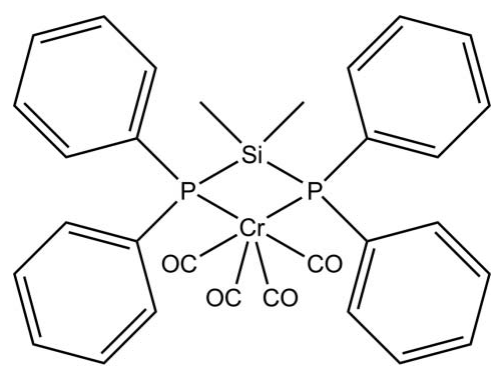

\section{Experimental}

Crystal data

$\left[\mathrm{Cr}\left(\mathrm{C}_{26} \mathrm{H}_{26} \mathrm{P}_{2} \mathrm{Si}\right)(\mathrm{CO})_{4}\right]$

$M_{r}=592.54$

Monoclinic, $P 2_{1} / c$

$a=13.7832$ (4) $\AA$

$b=11.9204(2) \AA$

$c=18.1329(5) \AA$

$\beta=102.073(2)^{\circ}$

$V=2913.36(13) \AA^{3}$

$Z=4$

Mo $K \alpha$ radiation

$\mu=0.58 \mathrm{~mm}^{-1}$

$T=200 \mathrm{~K}$

$0.45 \times 0.40 \times 0.38 \mathrm{~mm}$

\section{Data collection}

Stoe IPDS II diffractometer

Absorption correction: numerical

$(X$-SHAPE and X-RED32; Stoe

\& Cie, 2005)

$T_{\min }=0.773, T_{\max }=0.867$

Refinement

$R\left[F^{2}>2 \sigma\left(F^{2}\right)\right]=0.026$

$w R\left(F^{2}\right)=0.065$

$S=0.93$

6688 reflections

345 parameters

47168 measured reflections 6688 independent reflections 5370 reflections with $I>2 \sigma(I)$ $R_{\text {int }}=0.032$

Data collection: $X$-AREA (Stoe \& Cie, 2005); cell refinement: $X$-AREA; data reduction: $X$-AREA; program(s) used to solve structure: SHELXS97 (Sheldrick, 2008); program(s) used to refine structure: SHELXL97 (Sheldrick, 2008); molecular graphics: XP in SHELXTL (Sheldrick, 2008); software used to prepare material for publication: SHELXL97.

This work was supported by the Leibniz-Institut für Katalyse e. V. an der Universität Rostock.

Supplementary data and figures for this paper are available from the IUCr electronic archives (Reference: CV2781).

\section{References}

Chen, T., Jackson, J., Jasper, S. A., Duesler, E. N., Nöth, H. \& Paine, R. T. (1999). J. Organomet. Chem. 582, 25-31.

Hassler, K. \& Seidl, S. (1988). Monatsh. Chem. 119, 1241-1244.

Sheldrick, G. M. (2008). Acta Cryst. A64, 112-122.

Sheldrick, W. S. \& Borkenstein, A. (1977). Acta Cryst. B33, 2916-2918.

Stoe \& Cie (2005). X-SHAPE, X-RED32 and X-AREA. Stoe \& Cie, Darmstadt, Germany. 


\section{supporting information}

Acta Cryst. (2010). E66, m1494 [https://doi.org/10.1107/S1600536810043679]

[Bis(diphenylphosphanyl)dimethylsilane- $\left.\kappa^{2} P, P^{\prime}\right]$ tetracarbonylchromium(0)

Normen Peulecke, Stephan Peitz, Bernd H. Müller, Anke Spannenberg and Uwe Rosenthal

\section{S1. Comment}

Disphosphines are widely used as chelate ligands for complex formation. Among them the silicon-bridged species are not common. There are only few examples of structurally characterized complexes with a $\mathrm{SiMe}_{2}$ bridge (Chen et al., 1999) or $\mathrm{SiMe}_{2} \mathrm{SiMe}_{2}$ bridge (Sheldrick \& Borkenstein, 1977). In the present publication, we report on the formation and molecular structure of the title compound, which was observed to be the single product of a complex formation of $\mathrm{Ph}_{2} \mathrm{PSiMe}_{2} \mathrm{PPh}_{2}$ with $\mathrm{Cr}(\mathrm{CO})_{6}$. The synthesis of the starting ligand was already reported by Hassler \& Seidl (1988).

In the molecular structure of the title complex the chelating disphosphine and four carbonyl ligands are coordinated to the $\mathrm{Cr}$ atom (Fig. 1). The coordination geometry at the metal center is best described as distorted octahedral. The observed bite angle $\mathrm{P}-\mathrm{Cr}-\mathrm{P}$ is $80.27(1)^{\circ}$ and the $\mathrm{P}-\mathrm{Si}-\mathrm{P}$ angle of the complexed ligand is $85.31(2)^{\circ}$.

\section{S2. Experimental}

$\mathrm{Cr}(\mathrm{CO})_{6}(175 \mathrm{mg}, 0.8 \mathrm{mmol})$ was added to a solution of $\mathrm{Ph}_{2} \mathrm{PSiMe}_{2} \mathrm{PPh}_{2}(321 \mathrm{mg}, 0.75 \mathrm{mmol})$ in $20 \mathrm{ml}$ of toluene and the resulting solution was stirred at reflux temperature for $72 \mathrm{~h}$. Subsequently, the formed yellow solution was cooled down to $0^{\circ} \mathrm{C}$ and filtered. Toluene was removed in vacuum and the product was extracted with dichloromethane. The major part of dichloromethane was removed and the remaining solution was over-layered with $n$-hexane to get single crystals of the title compound. The yellow compound was fully characterized by standard analytical methods e.g. ${ }^{31} \mathrm{P} \mathrm{NMR}:\left(\mathrm{CD}_{2} \mathrm{Cl}_{2}\right)$ : 5.9 p.p.m.

\section{S3. Refinement}

All $\mathrm{H}$ atoms were placed in idealized positions with $\mathrm{d}(\mathrm{C}-\mathrm{H})=0.98\left(\mathrm{CH}_{3}\right)$ and $0.95 \AA(\mathrm{CH})$ and refined using a riding model with $U_{\text {iso }}(\mathrm{H})$ fixed at $1.5 U_{\text {eq }}(\mathrm{C})$ for $\mathrm{CH}_{3}$ and $1.2 U_{\text {eq }}(\mathrm{C})$ for $\mathrm{CH}$. 


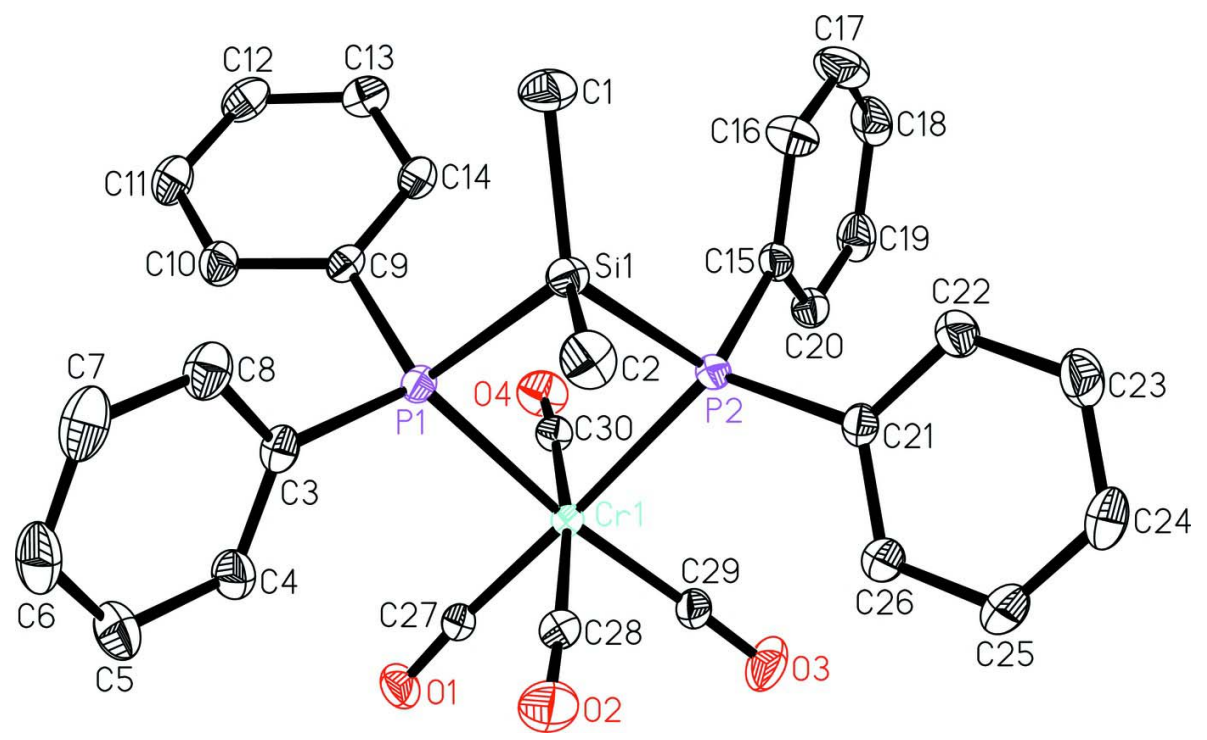

Figure 1

The molecular structure of the title compound showing the atom-labelling scheme. $\mathrm{H}$ atoms are omitted for clarity. Displacement ellipsoids are drawn at the $30 \%$ probability level.

[Bis(diphenylphosphanyl)dimethylsilane- $\left.\boldsymbol{\kappa}^{2} \boldsymbol{P}, \boldsymbol{P}^{\prime}\right]$ tetracarbonylchromium

\section{Crystal data}

$\left[\mathrm{Cr}\left(\mathrm{C}_{26} \mathrm{H}_{26} \mathrm{P}_{2} \mathrm{Si}\right)(\mathrm{CO})_{4}\right]$

$M_{r}=592.54$

Monoclinic, $P 2_{1} / c$

Hall symbol: -P 2ybc

$a=13.7832$ (4) $\AA$

$b=11.9204$ (2) $\AA$

$c=18.1329(5) \AA$

$\beta=102.073(2)^{\circ}$

$V=2913.36(13) \AA^{3}$

$Z=4$

\section{Data collection}

Stoe IPDS II

\section{diffractometer}

Radiation source: fine-focus sealed tube

Graphite monochromator

$\omega$ scans

Absorption correction: numerical

( $X$-SHAPE and $X$-RED32; Stoe \& Cie, 2005)

$T_{\min }=0.773, T_{\max }=0.867$

\section{Refinement}

Refinement on $F^{2}$

Least-squares matrix: full

$R\left[F^{2}>2 \sigma\left(F^{2}\right)\right]=0.026$

$w R\left(F^{2}\right)=0.065$

$S=0.93$

6688 reflections

345 parameters
$F(000)=1224$

$D_{\mathrm{x}}=1.351 \mathrm{Mg} \mathrm{m}^{-3}$

Mo $K \alpha$ radiation, $\lambda=0.71073 \AA$

Cell parameters from 13232 reflections

$\theta=2.0-29.6^{\circ}$

$\mu=0.58 \mathrm{~mm}^{-1}$

$T=200 \mathrm{~K}$

Prism, yellow

$0.45 \times 0.40 \times 0.38 \mathrm{~mm}$

47168 measured reflections

6688 independent reflections

5370 reflections with $I>2 \sigma(I)$

$R_{\text {int }}=0.032$

$\theta_{\max }=27.5^{\circ}, \theta_{\min }=2.1^{\circ}$

$h=-17 \rightarrow 17$

$k=-15 \rightarrow 15$

$l=-23 \rightarrow 23$

6 restraints

Primary atom site location: structure-invariant direct methods

Secondary atom site location: difference Fourier map

Hydrogen site location: inferred from neighbouring sites 
H-atom parameters constrained

$w=1 /\left[\sigma^{2}\left(F_{\mathrm{o}}^{2}\right)+(0.0437 P)^{2}\right]$

where $P=\left(F_{\mathrm{o}}^{2}+2 F_{\mathrm{c}}{ }^{2}\right) / 3$

$$
\begin{aligned}
& (\Delta / \sigma)_{\max }=0.001 \\
& \Delta \rho_{\max }=0.32 \mathrm{e} \AA^{-3} \\
& \Delta \rho_{\min }=-0.33 \mathrm{e}^{-3}
\end{aligned}
$$

Special details

Geometry. All e.s.d.'s (except the e.s.d. in the dihedral angle between two l.s. planes) are estimated using the full covariance matrix. The cell e.s.d.'s are taken into account individually in the estimation of e.s.d.'s in distances, angles and torsion angles; correlations between e.s.d.'s in cell parameters are only used when they are defined by crystal symmetry. An approximate (isotropic) treatment of cell e.s.d.'s is used for estimating e.s.d.'s involving 1.s. planes.

Refinement. Refinement of $F^{2}$ against ALL reflections. The weighted $R$-factor $w R$ and goodness of fit $S$ are based on $F^{2}$, conventional $R$-factors $R$ are based on $F$, with $F$ set to zero for negative $F^{2}$. The threshold expression of $F^{2}>\sigma\left(F^{2}\right)$ is used only for calculating $R$-factors $(\mathrm{gt})$ etc. and is not relevant to the choice of reflections for refinement. $R$-factors based on $F^{2}$

\begin{tabular}{|c|c|c|c|c|}
\hline & $x$ & $y$ & $z$ & $U_{\text {iso }} * / U_{\text {eq }}$ \\
\hline $\mathrm{C} 1$ & $0.25499(13)$ & $0.34671(15)$ & $0.23882(9)$ & $0.0452(4)$ \\
\hline $\mathrm{H} 1 \mathrm{~A}$ & 0.2964 & 0.3105 & 0.2079 & $0.068 *$ \\
\hline H1B & 0.2941 & 0.4035 & 0.2711 & $0.068^{*}$ \\
\hline $\mathrm{H} 1 \mathrm{C}$ & 0.1979 & 0.3826 & 0.2060 & $0.068 *$ \\
\hline $\mathrm{C} 2$ & $0.13677(12)$ & $0.12988(15)$ & $0.23902(10)$ & $0.0445(4)$ \\
\hline $\mathrm{H} 2 \mathrm{~A}$ & 0.0795 & 0.1647 & 0.2057 & $0.067^{*}$ \\
\hline $\mathrm{H} 2 \mathrm{~B}$ & 0.1136 & 0.0746 & 0.2714 & $0.067 *$ \\
\hline $\mathrm{H} 2 \mathrm{C}$ & 0.1782 & 0.0926 & 0.2086 & $0.067^{*}$ \\
\hline $\mathrm{C} 3$ & $0.37225(9)$ & $0.03094(13)$ & $0.35878(8)$ & $0.0312(3)$ \\
\hline $\mathrm{C} 4$ & $0.38402(12)$ & $-0.06394(13)$ & $0.40336(10)$ & $0.0411(4)$ \\
\hline $\mathrm{H} 4$ & 0.3647 & -0.0619 & 0.4507 & $0.049 *$ \\
\hline $\mathrm{C} 5$ & $0.42314(14)$ & $-0.16177(14)$ & $0.38092(10)$ & $0.0525(4)$ \\
\hline H5 & 0.4309 & -0.2259 & 0.4127 & $0.063^{*}$ \\
\hline C6 & $0.45082(13)$ & $-0.16610(15)$ & $0.31233(9)$ & $0.0535(5)$ \\
\hline H6 & 0.4776 & -0.2332 & 0.2965 & $0.064 *$ \\
\hline $\mathrm{C} 7$ & $0.43945(13)$ & $-0.07262(14)$ & $0.26685(10)$ & $0.0553(5)$ \\
\hline $\mathrm{H7}$ & 0.4588 & -0.0753 & 0.2196 & $0.066^{*}$ \\
\hline $\mathrm{C} 8$ & $0.40018(12)$ & $0.02518(15)$ & $0.28928(9)$ & $0.0442(4)$ \\
\hline H8 & 0.3922 & 0.0889 & 0.2571 & $0.053^{*}$ \\
\hline C9 & $0.43399(9)$ & $0.25333(12)$ & $0.40684(7)$ & $0.0277(3)$ \\
\hline $\mathrm{C} 10$ & $0.52916(10)$ & $0.20972(14)$ & $0.41518(9)$ & 0.0378 \\
\hline H10 & 0.5384 & 0.1311 & 0.4113 & $0.045^{*}$ \\
\hline C11 & $0.61087(11)$ & $0.28078(16)$ & $0.42918(10)$ & $0.0455(4)$ \\
\hline H11 & 0.6758 & 0.2503 & 0.4358 & $0.055^{*}$ \\
\hline $\mathrm{C} 12$ & $0.59842(12)$ & $0.39468(16)$ & $0.43350(9)$ & $0.0438(4)$ \\
\hline H12 & 0.6546 & 0.4428 & 0.4432 & $0.053 *$ \\
\hline $\mathrm{C} 13$ & $0.50415(12)$ & $0.43899(14)$ & $0.42375(9)$ & 0.0386 \\
\hline H13 & 0.4952 & 0.5179 & 0.4251 & $0.046^{*}$ \\
\hline C14 & $0.42244(10)$ & $0.36853(13)$ & $0.41193(8)$ & 0.0330 \\
\hline H14 & 0.3579 & 0.3994 & 0.4073 & $0.040 *$ \\
\hline C15 & $0.13366(9)$ & $0.43218(12)$ & $0.41500(8)$ & $0.0302(3)$ \\
\hline C16 & $0.14302(13)$ & $0.51650(14)$ & $0.36418(10)$ & $0.0456(4)$ \\
\hline
\end{tabular}
are statistically about twice as large as those based on $F$, and $R$-factors based on ALL data will be even larger.

Fractional atomic coordinates and isotropic or equivalent isotropic displacement parameters $\left(\AA^{2}\right)$ 


\begin{tabular}{|c|c|c|c|c|}
\hline H16 & 0.1494 & 0.4976 & 0.3145 & $0.055^{*}$ \\
\hline $\mathrm{C} 17$ & $0.14317(15)$ & $0.62837(15)$ & $0.38556(12)$ & $0.0561(5)$ \\
\hline H17 & 0.1496 & 0.6854 & 0.3503 & $0.067 *$ \\
\hline $\mathrm{C} 18$ & $0.13416(12)$ & $0.65736(14)$ & $0.45713(12)$ & $0.0493(4)$ \\
\hline H18 & 0.1344 & 0.7341 & 0.4714 & $0.059 *$ \\
\hline C19 & $0.12477(12)$ & $0.57488(15)$ & $0.50772(10)$ & $0.0446(4)$ \\
\hline H19 & 0.1183 & 0.5946 & 0.5572 & $0.053 *$ \\
\hline $\mathrm{C} 20$ & $0.12470(10)$ & $0.46281(13)$ & $0.48722(9)$ & $0.0355(3)$ \\
\hline $\mathrm{H} 20$ & 0.1185 & 0.4064 & 0.5229 & $0.043 *$ \\
\hline $\mathrm{C} 21$ & $-0.00463(9)$ & $0.25993(12)$ & $0.35370(8)$ & $0.0291(3)$ \\
\hline $\mathrm{C} 22$ & $-0.06017(11)$ & $0.33304(14)$ & $0.30152(9)$ & $0.0394(3)$ \\
\hline $\mathrm{H} 22$ & -0.0289 & 0.3955 & 0.2837 & $0.047^{*}$ \\
\hline $\mathrm{C} 23$ & $-0.16100(12)$ & $0.31502(16)$ & $0.27540(10)$ & $0.0466(4)$ \\
\hline $\mathrm{H} 23$ & -0.1985 & 0.3656 & 0.2401 & $0.056^{*}$ \\
\hline $\mathrm{C} 24$ & $-0.20685(11)$ & $0.22462(16)$ & $0.30030(10)$ & $0.0466(4)$ \\
\hline $\mathrm{H} 24$ & -0.2760 & 0.2130 & 0.2825 & $0.056^{*}$ \\
\hline $\mathrm{C} 25$ & -0.15301 & $0.15113(16)$ & $0.35085(11)$ & $0.0479(4)$ \\
\hline $\mathrm{H} 25$ & -0.1847 & 0.0880 & 0.3675 & $0.058 *$ \\
\hline $\mathrm{C} 26$ & $-0.05175(11)$ & $0.16869(14)$ & $0.37797(9)$ & $0.0392(3)$ \\
\hline $\mathrm{H} 26$ & -0.0149 & 0.1177 & 0.4133 & $0.047^{*}$ \\
\hline $\mathrm{C} 27$ & $0.29769(10)$ & 0.06277 (12) & $0.55229(8)$ & $0.0303(3)$ \\
\hline $\mathrm{C} 28$ & $0.16332(10)$ & $0.02616(13)$ & $0.43194(9)$ & $0.0353(3)$ \\
\hline $\mathrm{C} 29$ & $0.12597(10)$ & $0.16240(13)$ & $0.54054(8)$ & $0.0340(3)$ \\
\hline $\mathrm{C} 30$ & $0.29387(10)$ & $0.27186(13)$ & $0.53968(8)$ & $0.0318(3)$ \\
\hline $\mathrm{Cr} 1$ & $0.221253(14)$ & $0.156127(18)$ & $0.482132(12)$ & $0.02433(6)$ \\
\hline $\mathrm{O} 1$ & $0.34093(8)$ & $0.00645(10)$ & $0.59947(6)$ & $0.0440(3)$ \\
\hline $\mathrm{O} 2$ & $0.13246(10)$ & $-0.05665(11)$ & $0.40470(8)$ & $0.0575(3)$ \\
\hline $\mathrm{O} 3$ & $0.06848(9)$ & $0.16388(12)$ & $0.57808(7)$ & $0.0554(3)$ \\
\hline $\mathrm{O} 4$ & $0.34046(9)$ & $0.33533(10)$ & $0.57916(7)$ & $0.0500(3)$ \\
\hline P1 & $0.32570(2)$ & $0.16071(3)$ & 0.392418 (19) & $0.02575(8)$ \\
\hline P2 & $0.12811(2)$ & $0.28288(3)$ & $0.39145(2)$ & $0.02589(8)$ \\
\hline Si1 & $0.21062(3)$ & $0.23959(3)$ & $0.29856(2)$ & $0.02921(9)$ \\
\hline
\end{tabular}

Atomic displacement parameters $\left(\AA^{2}\right)$

\begin{tabular}{lllllll}
\hline & $U^{11}$ & $U^{22}$ & $U^{33}$ & $U^{12}$ & $U^{13}$ & $U^{23}$ \\
\hline C1 & $0.0530(9)$ & $0.0510(10)$ & $0.0323(8)$ & $-0.0054(8)$ & $0.0102(7)$ & $0.0108(7)$ \\
C2 & $0.0419(8)$ & $0.0442(10)$ & $0.0423(9)$ & $-0.0046(7)$ & $-0.0029(7)$ & $-0.0077(7)$ \\
C3 & $0.0247(6)$ & $0.0372(8)$ & $0.0317(7)$ & $-0.0008(5)$ & $0.0059(5)$ & $-0.0067(6)$ \\
C4 & $0.0453(8)$ & $0.0373(9)$ & $0.0439(9)$ & $0.0068(7)$ & $0.0163(7)$ & $-0.0026(7)$ \\
C5 & $0.0559(10)$ & $0.0401(10)$ & $0.0636(12)$ & $0.0125(8)$ & $0.0175(9)$ & $-0.0059(9)$ \\
C6 & $0.0445(9)$ & $0.0551(12)$ & $0.0605(11)$ & $0.0092(8)$ & $0.0098(8)$ & $-0.0249(9)$ \\
C7 & $0.0479(9)$ & $0.0807(14)$ & $0.0400(9)$ & $0.0054(9)$ & $0.0151(7)$ & $-0.0227(10)$ \\
C8 & $0.0421(8)$ & $0.0581(11)$ & $0.0341(8)$ & $0.0032(7)$ & $0.0118(6)$ & $-0.0048(8)$ \\
C9 & $0.0248(6)$ & $0.0349(8)$ & $0.0238(6)$ & $-0.0035(5)$ & $0.0063(5)$ & $-0.0002(5)$ \\
C10 & $0.0285(7)$ & $0.0390(9)$ & $0.0449(9)$ & $0.0006(6)$ & $0.0058(6)$ & $0.0002(7)$ \\
C11 & $0.0256(7)$ & $0.0560(11)$ & $0.0543(10)$ & $-0.0031(7)$ & $0.0069(7)$ & $-0.0009(8)$ \\
C12 & $0.0358(8)$ & $0.0536(11)$ & $0.0423(9)$ & $-0.0163(7)$ & $0.0091(6)$ & $-0.0058(8)$
\end{tabular}




$\begin{array}{lllllll}\text { C13 } & 0.0454(8) & 0.0366(9) & 0.0355(8) & -0.0089(6) & 0.0121(6) & -0.0067(6) \\ \text { C14 } & 0.0309(7) & 0.0380(8) & 0.0313(7) & -0.0009(6) & 0.0089(5) & -0.0038(6) \\ \text { C15 } & 0.0233(6) & 0.0259(7) & 0.0396(8) & 0.0006(5) & 0.0024(5) & 0.0016(6) \\ \text { C16 } & 0.0587(10) & 0.0330(9) & 0.0449(9) & -0.0010(7) & 0.0102(8) & 0.0067(7) \\ \text { C17 } & 0.0694(12) & 0.0301(9) & 0.0673(13) & -0.0016(8) & 0.0110(10) & 0.0113(8) \\ \text { C18 } & 0.0420(9) & 0.0285(8) & 0.0741(13) & 0.0022(7) & 0.0047(8) & -0.0062(8) \\ \text { C19 } & 0.0377(8) & 0.0407(9) & 0.0547(10) & 0.0033(7) & 0.0084(7) & -0.0117(8) \\ \text { C20 } & 0.0326(7) & 0.0326(8) & 0.0420(8) & 0.0011(6) & 0.0090(6) & -0.0003(6) \\ \text { C21 } & 0.0233(6) & 0.0309(7) & 0.0327(7) & 0.0015(5) & 0.0050(5) & -0.0012(6) \\ \text { C22 } & 0.0331(7) & 0.0380(9) & 0.0441(9) & 0.0013(6) & 0.0012(6) & 0.0060(7) \\ \text { C23 } & 0.0339(8) & 0.0514(11) & 0.0486(10) & 0.0068(7) & -0.0049(7) & 0.0029(8) \\ \text { C24 } & 0.0256(7) & 0.0601(11) & 0.0510(9) & -0.0017(7) & 0.0015(6) & -0.0074(8) \\ \text { C25 } & 0.0309(7) & 0.0517(11) & 0.0605(11) & -0.0099(7) & 0.0080(7) & 0.0045(9) \\ \text { C26 } & 0.0295(7) & 0.0384(9) & 0.0481(9) & -0.0022(6) & 0.0044(6) & 0.0072(7) \\ \text { C27 } & 0.0280(6) & 0.0306(7) & 0.0345(7) & 0.0031(5) & 0.0119(5) & 0.0025(6) \\ \text { C28 } & 0.0300(7) & 0.0318(8) & 0.0452(8) & -0.0014(6) & 0.0104(6) & 0.0039(7) \\ \text { C29 } & 0.0270(6) & 0.0393(8) & 0.0356(7) & 0.0043(6) & 0.0064(6) & 0.0072(6) \\ \text { C30 } & 0.0320(7) & 0.0318(8) & 0.0325(7) & 0.0022(6) & 0.0085(6) & 0.0040(6) \\ \text { Cr1 } & 0.02209(10) & 0.02451(11) & 0.02716(11) & 0.00111(8) & 0.00691(7) & 0.00371(9) \\ \text { O1 } & 0.0445(6) & 0.0457(7) & 0.0418(6) & 0.0156(5) & 0.0093(5) & 0.0138(5) \\ \text { O2 } & 0.0552(7) & 0.0373(7) & 0.0805(9) & -0.0142(6) & 0.0154(7) & -0.0111(6) \\ \text { O3 } & 0.0383(6) & 0.0841(10) & 0.0499(7) & 0.0068(6) & 0.0230(5) & 0.0083(7) \\ \text { O4 } & 0.0540(7) & 0.0442(7) & 0.0476(7) & -0.0103(6) & 0.0007(5) & -0.0063(6) \\ \text { P1 } & 0.02266(15) & 0.02917(18) & 0.02576(16) & -0.00015(13) & 0.00579(12) & 0.00080(14) \\ \text { P2 } & 0.02295(15) & 0.02462(18) & 0.02950(17) & 0.00002(12) & 0.00413(12) & 0.00330(14) \\ \text { S11 } & 0.02813(18) & 0.0322(2) & 0.02609(18) & -0.00233(15) & 0.00286(14) & 0.00240(15) \\ & & & & & & \end{array}$

Geometric parameters $\left(A,{ }^{\circ}\right)$

\begin{tabular}{llll}
\hline $\mathrm{C} 1-\mathrm{Si} 1$ & $1.8588(16)$ & $\mathrm{C} 15-\mathrm{P} 2$ & $1.8281(15)$ \\
$\mathrm{C} 1-\mathrm{H} 1 \mathrm{~A}$ & 0.9800 & $\mathrm{C} 16-\mathrm{C} 17$ & $1.389(3)$ \\
$\mathrm{C} 1-\mathrm{H} 1 \mathrm{~B}$ & 0.9800 & $\mathrm{C} 16-\mathrm{H} 16$ & 0.9500 \\
$\mathrm{C} 1-\mathrm{H} 1 \mathrm{C}$ & 0.9800 & $\mathrm{C} 17-\mathrm{C} 18$ & $1.373(3)$ \\
$\mathrm{C} 2-\mathrm{S} 11$ & $\mathrm{C} 17-\mathrm{H} 17$ & 0.9500 \\
$\mathrm{C} 2-\mathrm{H} 2 \mathrm{~A}$ & $1.8580(16)$ & $\mathrm{C} 18-\mathrm{C} 19$ & $1.369(3)$ \\
$\mathrm{C} 2-\mathrm{H} 2 \mathrm{~B}$ & 0.9800 & $\mathrm{C} 18-\mathrm{H} 18$ & 0.9500 \\
$\mathrm{C} 2-\mathrm{H} 2 \mathrm{C}$ & 0.9800 & $\mathrm{C} 19-\mathrm{C} 20$ & $1.387(2)$ \\
$\mathrm{C} 3-\mathrm{C} 4$ & 0.9800 & $\mathrm{C} 19-\mathrm{H} 19$ & 0.9500 \\
$\mathrm{C} 3-\mathrm{C} 8$ & $1.380(2)$ & $\mathrm{C} 20-\mathrm{H} 20$ & 0.9500 \\
$\mathrm{C} 3-\mathrm{P} 1$ & $1.394(2)$ & $\mathrm{C} 21-\mathrm{C} 26$ & $1.385(2)$ \\
$\mathrm{C} 4-\mathrm{C} 5$ & $1.8277(15)$ & $\mathrm{C} 21-\mathrm{C} 22$ & $1.392(2)$ \\
$\mathrm{C} 4-\mathrm{H} 4$ & $1.3813(14)$ & $\mathrm{C} 21-\mathrm{P} 2$ & $1.8350(13)$ \\
$\mathrm{C} 5-\mathrm{C} 6$ & 0.9500 & $\mathrm{C} 22-\mathrm{C} 23$ & $1.388(2)$ \\
$\mathrm{C} 5-\mathrm{H} 5$ & $1.3760(15)$ & $\mathrm{C} 22-\mathrm{H} 22$ & 0.9500 \\
$\mathrm{C} 6-\mathrm{C} 7$ & 0.9500 & $\mathrm{C} 23-\mathrm{C} 24$ & $1.373(3)$ \\
$\mathrm{C} 6-\mathrm{H} 6$ & $1.3756(15)$ & $\mathrm{C} 23-\mathrm{H} 23$ & 0.9500 \\
$\mathrm{C} 7-\mathrm{C} 8$ & 0.9500 & $\mathrm{C} 24-\mathrm{C} 25$ & $1.369(3)$ \\
$\mathrm{C} 7-\mathrm{H} 7$ & $1.3818(15)$ & $\mathrm{C} 24-\mathrm{H} 24$ & 0.9500
\end{tabular}




\begin{tabular}{|c|c|c|c|}
\hline $\mathrm{C} 8-\mathrm{H} 8$ & 0.9500 & $\mathrm{C} 25-\mathrm{C} 26$ & $1.395(2)$ \\
\hline $\mathrm{C} 9-\mathrm{C} 14$ & $1.388(2)$ & $\mathrm{C} 25-\mathrm{H} 25$ & 0.9500 \\
\hline $\mathrm{C} 9-\mathrm{C} 10$ & $1.3896(19)$ & $\mathrm{C} 26-\mathrm{H} 26$ & 0.9500 \\
\hline C9-P1 & $1.8308(13)$ & $\mathrm{C} 27-\mathrm{O} 1$ & $1.1509(17)$ \\
\hline $\mathrm{C} 10-\mathrm{C} 11$ & $1.389(2)$ & $\mathrm{C} 27-\mathrm{Cr} 1$ & $1.8455(14)$ \\
\hline $\mathrm{C} 10-\mathrm{H} 10$ & 0.9500 & $\mathrm{C} 28-\mathrm{O} 2$ & $1.1452(19)$ \\
\hline $\mathrm{C} 11-\mathrm{C} 12$ & $1.373(3)$ & $\mathrm{C} 28-\mathrm{Cr} 1$ & $1.8874(16)$ \\
\hline $\mathrm{C} 11-\mathrm{H} 11$ & 0.9500 & $\mathrm{C} 29-\mathrm{O} 3$ & $1.1477(18)$ \\
\hline $\mathrm{C} 12-\mathrm{C} 13$ & $1.379(2)$ & $\mathrm{C} 29-\mathrm{Cr} 1$ & $1.8530(14)$ \\
\hline $\mathrm{C} 12-\mathrm{H} 12$ & 0.9500 & $\mathrm{C} 30-\mathrm{O} 4$ & $1.1424(18)$ \\
\hline $\mathrm{C} 13-\mathrm{C} 14$ & $1.385(2)$ & $\mathrm{C} 30-\mathrm{Cr} 1$ & $1.8860(15)$ \\
\hline C13-H13 & 0.9500 & $\mathrm{Cr} 1-\mathrm{P} 1$ & $2.3877(4)$ \\
\hline $\mathrm{C} 14-\mathrm{H} 14$ & 0.9500 & $\mathrm{Cr} 1-\mathrm{P} 2$ & $2.3983(4)$ \\
\hline $\mathrm{C} 15-\mathrm{C} 16$ & $1.388(2)$ & P1—Si1 & $2.2731(5)$ \\
\hline $\mathrm{C} 15-\mathrm{C} 20$ & $1.390(2)$ & $\mathrm{P} 2-\mathrm{Si} 1$ & $2.2798(5)$ \\
\hline $\mathrm{Si} 1-\mathrm{C} 1-\mathrm{H} 1 \mathrm{~A}$ & 109.5 & $\mathrm{C} 18-\mathrm{C} 19-\mathrm{H} 19$ & 119.8 \\
\hline $\mathrm{Si} 1-\mathrm{C} 1-\mathrm{H} 1 \mathrm{~B}$ & 109.5 & $\mathrm{C} 20-\mathrm{C} 19-\mathrm{H} 19$ & 119.8 \\
\hline $\mathrm{H} 1 \mathrm{~A}-\mathrm{C} 1-\mathrm{H} 1 \mathrm{~B}$ & 109.5 & $\mathrm{C} 19-\mathrm{C} 20-\mathrm{C} 15$ & $120.70(15)$ \\
\hline $\mathrm{Si} 1-\mathrm{C} 1-\mathrm{H} 1 \mathrm{C}$ & 109.5 & $\mathrm{C} 19-\mathrm{C} 20-\mathrm{H} 20$ & 119.7 \\
\hline $\mathrm{H} 1 \mathrm{~A}-\mathrm{C} 1-\mathrm{H} 1 \mathrm{C}$ & 109.5 & $\mathrm{C} 15-\mathrm{C} 20-\mathrm{H} 20$ & 119.7 \\
\hline $\mathrm{H} 1 \mathrm{~B}-\mathrm{C} 1-\mathrm{H} 1 \mathrm{C}$ & 109.5 & $\mathrm{C} 26-\mathrm{C} 21-\mathrm{C} 22$ & $118.69(13)$ \\
\hline $\mathrm{Si} 1-\mathrm{C} 2-\mathrm{H} 2 \mathrm{~A}$ & 109.5 & $\mathrm{C} 26-\mathrm{C} 21-\mathrm{P} 2$ & $119.72(11)$ \\
\hline $\mathrm{Si} 1-\mathrm{C} 2-\mathrm{H} 2 \mathrm{~B}$ & 109.5 & $\mathrm{C} 22-\mathrm{C} 21-\mathrm{P} 2$ & $121.59(11)$ \\
\hline $\mathrm{H} 2 \mathrm{~A}-\mathrm{C} 2-\mathrm{H} 2 \mathrm{~B}$ & 109.5 & $\mathrm{C} 23-\mathrm{C} 22-\mathrm{C} 21$ & $120.26(15)$ \\
\hline $\mathrm{Si} 1-\mathrm{C} 2-\mathrm{H} 2 \mathrm{C}$ & 109.5 & $\mathrm{C} 23-\mathrm{C} 22-\mathrm{H} 22$ & 119.9 \\
\hline $\mathrm{H} 2 \mathrm{~A}-\mathrm{C} 2-\mathrm{H} 2 \mathrm{C}$ & 109.5 & $\mathrm{C} 21-\mathrm{C} 22-\mathrm{H} 22$ & 119.9 \\
\hline $\mathrm{H} 2 \mathrm{~B}-\mathrm{C} 2-\mathrm{H} 2 \mathrm{C}$ & 109.5 & $\mathrm{C} 24-\mathrm{C} 23-\mathrm{C} 22$ & $120.42(15)$ \\
\hline $\mathrm{C} 4-\mathrm{C} 3-\mathrm{C} 8$ & $117.93(14)$ & $\mathrm{C} 24-\mathrm{C} 23-\mathrm{H} 23$ & 119.8 \\
\hline $\mathrm{C} 4-\mathrm{C} 3-\mathrm{P} 1$ & $120.31(11)$ & $\mathrm{C} 22-\mathrm{C} 23-\mathrm{H} 23$ & 119.8 \\
\hline $\mathrm{C} 8-\mathrm{C} 3-\mathrm{P} 1$ & $121.72(12)$ & $\mathrm{C} 25-\mathrm{C} 24-\mathrm{C} 23$ & $120.02(14)$ \\
\hline $\mathrm{C} 3-\mathrm{C} 4-\mathrm{C} 5$ & $121.64(15)$ & $\mathrm{C} 25-\mathrm{C} 24-\mathrm{H} 24$ & 120.0 \\
\hline $\mathrm{C} 3-\mathrm{C} 4-\mathrm{H} 4$ & 119.2 & $\mathrm{C} 23-\mathrm{C} 24-\mathrm{H} 24$ & 120.0 \\
\hline $\mathrm{C} 5-\mathrm{C} 4-\mathrm{H} 4$ & 119.2 & $\mathrm{C} 24-\mathrm{C} 25-\mathrm{C} 26$ & $120.15(16)$ \\
\hline $\mathrm{C} 6-\mathrm{C} 5-\mathrm{C} 4$ & $119.81(17)$ & $\mathrm{C} 24-\mathrm{C} 25-\mathrm{H} 25$ & 119.9 \\
\hline $\mathrm{C} 6-\mathrm{C} 5-\mathrm{H} 5$ & 120.1 & $\mathrm{C} 26-\mathrm{C} 25-\mathrm{H} 25$ & 119.9 \\
\hline $\mathrm{C} 4-\mathrm{C} 5-\mathrm{H} 5$ & 120.1 & $\mathrm{C} 21-\mathrm{C} 26-\mathrm{C} 25$ & $120.45(15)$ \\
\hline $\mathrm{C} 7-\mathrm{C} 6-\mathrm{C} 5$ & $119.55(16)$ & $\mathrm{C} 21-\mathrm{C} 26-\mathrm{H} 26$ & 119.8 \\
\hline $\mathrm{C} 7-\mathrm{C} 6-\mathrm{H} 6$ & 120.2 & $\mathrm{C} 25-\mathrm{C} 26-\mathrm{H} 26$ & 119.8 \\
\hline $\mathrm{C} 5-\mathrm{C} 6-\mathrm{H} 6$ & 120.2 & $\mathrm{O} 1-\mathrm{C} 27-\mathrm{Cr} 1$ & $175.10(12)$ \\
\hline $\mathrm{C} 6-\mathrm{C} 7-\mathrm{C} 8$ & $120.63(16)$ & $\mathrm{O} 2-\mathrm{C} 28-\mathrm{Cr} 1$ & $175.62(14)$ \\
\hline $\mathrm{C} 6-\mathrm{C} 7-\mathrm{H} 7$ & 119.7 & $\mathrm{O} 3-\mathrm{C} 29-\mathrm{Cr} 1$ & $177.97(14)$ \\
\hline $\mathrm{C} 8-\mathrm{C} 7-\mathrm{H} 7$ & 119.7 & $\mathrm{O} 4-\mathrm{C} 30-\mathrm{Cr} 1$ & $174.21(13)$ \\
\hline $\mathrm{C} 7-\mathrm{C} 8-\mathrm{C} 3$ & $120.44(16)$ & $\mathrm{C} 27-\mathrm{Cr} 1-\mathrm{C} 29$ & $90.06(6)$ \\
\hline $\mathrm{C} 7-\mathrm{C} 8-\mathrm{H} 8$ & 119.8 & $\mathrm{C} 27-\mathrm{Cr} 1-\mathrm{C} 30$ & $84.22(6)$ \\
\hline $\mathrm{C} 3-\mathrm{C} 8-\mathrm{H} 8$ & 119.8 & $\mathrm{C} 29-\mathrm{Cr} 1-\mathrm{C} 30$ & $90.67(6)$ \\
\hline $\mathrm{C} 14-\mathrm{C} 9-\mathrm{C} 10$ & $118.80(13)$ & $\mathrm{C} 27-\mathrm{Cr} 1-\mathrm{C} 28$ & $87.73(7)$ \\
\hline $\mathrm{C} 14-\mathrm{C} 9-\mathrm{P} 1$ & $120.32(10)$ & $\mathrm{C} 29-\mathrm{Cr} 1-\mathrm{C} 28$ & $91.71(7)$ \\
\hline
\end{tabular}




\begin{tabular}{|c|c|c|c|}
\hline $\mathrm{C} 10-\mathrm{C} 9-\mathrm{P} 1$ & $120.85(11)$ & $\mathrm{C} 30-\mathrm{Cr} 1-\mathrm{C} 28$ & $171.61(6)$ \\
\hline $\mathrm{C} 11-\mathrm{C} 10-\mathrm{C} 9$ & $120.16(15)$ & $\mathrm{C} 27-\mathrm{Cr} 1-\mathrm{P} 1$ & $98.33(4)$ \\
\hline $\mathrm{C} 11-\mathrm{C} 10-\mathrm{H} 10$ & 119.9 & $\mathrm{C} 29-\mathrm{Cr} 1-\mathrm{P} 1$ & $171.42(4)$ \\
\hline $\mathrm{C} 9-\mathrm{C} 10-\mathrm{H} 10$ & 119.9 & $\mathrm{C} 30-\mathrm{Cr} 1-\mathrm{P} 1$ & $91.97(4)$ \\
\hline $\mathrm{C} 12-\mathrm{C} 11-\mathrm{C} 10$ & $120.47(15)$ & $\mathrm{C} 28-\mathrm{Cr} 1-\mathrm{P} 1$ & $86.83(5)$ \\
\hline $\mathrm{C} 12-\mathrm{C} 11-\mathrm{H} 11$ & 119.8 & $\mathrm{C} 27-\mathrm{Cr} 1-\mathrm{P} 2$ & $177.47(5)$ \\
\hline $\mathrm{C} 10-\mathrm{C} 11-\mathrm{H} 11$ & 119.8 & $\mathrm{C} 29-\mathrm{Cr} 1-\mathrm{P} 2$ & $91.42(4)$ \\
\hline $\mathrm{C} 11-\mathrm{C} 12-\mathrm{C} 13$ & $119.81(14)$ & $\mathrm{C} 30-\mathrm{Cr} 1-\mathrm{P} 2$ & $93.70(4)$ \\
\hline $\mathrm{C} 11-\mathrm{C} 12-\mathrm{H} 12$ & 120.1 & $\mathrm{C} 28-\mathrm{Cr} 1-\mathrm{P} 2$ & $94.28(5)$ \\
\hline $\mathrm{C} 13-\mathrm{C} 12-\mathrm{H} 12$ & 120.1 & $\mathrm{P} 1-\mathrm{Cr} 1-\mathrm{P} 2$ & 80.270 (13) \\
\hline $\mathrm{C} 12-\mathrm{C} 13-\mathrm{C} 14$ & $120.08(15)$ & $\mathrm{C} 3-\mathrm{P} 1-\mathrm{C} 9$ & $103.03(6)$ \\
\hline $\mathrm{C} 12-\mathrm{C} 13-\mathrm{H} 13$ & 120.0 & $\mathrm{C} 3-\mathrm{P} 1-\mathrm{Si} 1$ & $109.81(5)$ \\
\hline $\mathrm{C} 14-\mathrm{C} 13-\mathrm{H} 13$ & 120.0 & C9-P1-Si1 & $106.13(5)$ \\
\hline $\mathrm{C} 13-\mathrm{C} 14-\mathrm{C} 9$ & $120.63(14)$ & $\mathrm{C} 3-\mathrm{P} 1-\mathrm{Cr} 1$ & $120.78(5)$ \\
\hline $\mathrm{C} 13-\mathrm{C} 14-\mathrm{H} 14$ & 119.7 & $\mathrm{C} 9-\mathrm{P} 1-\mathrm{Cr} 1$ & $120.41(4)$ \\
\hline $\mathrm{C} 9-\mathrm{C} 14-\mathrm{H} 14$ & 119.7 & $\mathrm{Si} 1-\mathrm{P} 1-\mathrm{Cr} 1$ & $95.386(16)$ \\
\hline $\mathrm{C} 16-\mathrm{C} 15-\mathrm{C} 20$ & $118.32(14)$ & $\mathrm{C} 15-\mathrm{P} 2-\mathrm{C} 21$ & $102.83(6)$ \\
\hline $\mathrm{C} 16-\mathrm{C} 15-\mathrm{P} 2$ & $123.72(12)$ & $\mathrm{C} 15-\mathrm{P} 2-\mathrm{Si} 1$ & $112.95(5)$ \\
\hline $\mathrm{C} 20-\mathrm{C} 15-\mathrm{P} 2$ & $117.92(11)$ & $\mathrm{C} 21-\mathrm{P} 2-\mathrm{Si} 1$ & $107.65(5)$ \\
\hline $\mathrm{C} 15-\mathrm{C} 16-\mathrm{C} 17$ & $120.34(17)$ & $\mathrm{C} 15-\mathrm{P} 2-\mathrm{Cr} 1$ & $117.58(5)$ \\
\hline $\mathrm{C} 15-\mathrm{C} 16-\mathrm{H} 16$ & 119.8 & $\mathrm{C} 21-\mathrm{P} 2-\mathrm{Cr} 1$ & $120.72(5)$ \\
\hline $\mathrm{C} 17-\mathrm{C} 16-\mathrm{H} 16$ & 119.8 & $\mathrm{Si} 1-\mathrm{P} 2-\mathrm{Cr} 1$ & 94.919 (16) \\
\hline $\mathrm{C} 18-\mathrm{C} 17-\mathrm{C} 16$ & $120.68(17)$ & $\mathrm{C} 2-\mathrm{Si} 1-\mathrm{C} 1$ & $110.61(8)$ \\
\hline $\mathrm{C} 18-\mathrm{C} 17-\mathrm{H} 17$ & 119.7 & $\mathrm{C} 2-\mathrm{Si} 1-\mathrm{P} 1$ & $110.83(6)$ \\
\hline $\mathrm{C} 16-\mathrm{C} 17-\mathrm{H} 17$ & 119.7 & $\mathrm{C} 1-\mathrm{Si} 1-\mathrm{P} 1$ & $117.22(6)$ \\
\hline $\mathrm{C} 19-\mathrm{C} 18-\mathrm{C} 17$ & $119.50(16)$ & $\mathrm{C} 2-\mathrm{Si} 1-\mathrm{P} 2$ & $106.93(6)$ \\
\hline $\mathrm{C} 19-\mathrm{C} 18-\mathrm{H} 18$ & 120.3 & $\mathrm{C} 1-\mathrm{Si} 1-\mathrm{P} 2$ & $123.42(6)$ \\
\hline $\mathrm{C} 17-\mathrm{C} 18-\mathrm{H} 18$ & 120.3 & $\mathrm{P} 1-\mathrm{Si} 1-\mathrm{P} 2$ & $85.312(17)$ \\
\hline $\mathrm{C} 18-\mathrm{C} 19-\mathrm{C} 20$ & $120.47(17)$ & & \\
\hline
\end{tabular}

